\title{
Bundle Branch Re-Entrant Ventricular Tachycardia by ECG Finding
}

National Cancer Institute

\section{Source}

National Cancer Institute. Bundle Branch Re-Entrant Ventricular Tachycardia by ECG

Finding. NCI Thesaurus. Code C71062.

An electrocardiog raphic finding of ventricular tachycardia incorporating both bundle branches into the reentry circuit. 